Investigación

\title{
CONOCIMIENTO DE LAS ENFERMERAS COMUNITARIAS SOBRE CUIDADOS DE LA ENFERMEDAD DE ALZHEIMER
}

\section{COMMUNITY HEALTH NURSING KNOWLEDGE ABOUT NURSING CARE IN THE ALZHEIMER DISEASES}

\section{Mabel Garzón Patterson}

Licenciada en Enfermería

Máster en Enfermería, Doctor en Ciencias de la Enfermería

Facultad de Enfermería Lidia Doce, Universidad de Ciencias Médicas de La Habana. La Habana, Cuba mabelgp@infomed.sld.cu

https://orcid.org/0000-0002-3751-4676

\section{Ricardo Izquierdo Medina}

Licenciado en Enfermería

Máster en Comunicación, Doctor en Ciencias Pedagógicas, Facultad de Ciencias Médicas 10 de Octubre, Universidad de Ciencias Médicas de La Habana. La Habana, Cuba rizdo@infomed.sld.cu https://orcid.org/0000-0003-0660-7252

\section{Yadira Pascual Cuesta}

Licenciada en Enfermería

Máster en Urgencias Médicas, Máster en Enfermería, Doctor en Ciencias de la Enfermería Facultad de Enfermería "Lidia Doce", Universidad de Ciencias Médicas de La Habana. La Habana, Cuba ypascual@infomed.sld.cu https://orcid.org/0000-0003-0372-9393

Artículo recibido el 21 de mayo de 2021. Aceptado en versión corregida el 24 de agosto de 2021.

\section{RESUMEN}

INTRODUCCIÓN: la demencia representa un alto costo social y económico; la enfermedad de Alzheimer la causa más frecuente, de ahí la importancia que los profesionales que intervienen en la atención y cuidado del paciente con enfermedad de Alzheimer y su cuidador dispongan de conocimientos y habilidades suficientes para su prevención, captación, diagnóstico inicial, seguimiento y tratamiento de los síntomas más comunes. OBJETIVO: identificar los conocimientos que tienen las enfermeras comunitarias sobre los cuidados en la enfermedad de Alzheimer. MÉTODOS: estudio descriptivo transversal en 20 enfermeras (os) que laboran en el Policlínico Docente "Julio Antonio Mella" del municipio Guanabacoa, durante enero y marzo del año 2018. Las variables estudiadas fueron: edad, sexo, años en el 
Conocimiento de las enfermeras comunitarias sobre cuidados...

ejercicio profesional, titulación y cuidados al cuidador de pacientes con enfermedad de Alzheimer. La información se obtuvo del cuestionario de conocimiento. Se utilizó la media, desviación estándar, frecuencia absoluta, porcentaje y el Alpha de Cronbach. RESULTADOS: La media de edad fue de 46,05 años. El 95,0\% de los participantes fueron del sexo femenino, el 75,0\% Licenciado en Enfermería, con un ejercicio profesional de 30 años y más (60.0\%); el 15,0\% no refieren cuidados a realizar al cuidador de pacientes con enfermedad de Alzheimer. CONCLUSIONES: las enfermeras comunitarias que laboran en el Policlínico Docente "Julio Antonio Mella" presentan escasos conocimientos sobre los cuidados a realizar al cuidador del paciente con enfermedad de Alzheimer.

Palabras clave: demencia; enfermedad de Alzheimer; personal de enfermería; cuidados de enfermería; enfermería en salud comunitaria; conocimiento.

\begin{abstract}
INTRODUCTION: dementia represents a high social and economic cost; the Alzheimer diseases is the most frequent cause, for this reason is important that the professionals that intervene in the attention and the patient's care with illness of Alzheimer and their caregiver have knowledge and enough abilities for their prevention, reception, initial diagnosis, monitoring and treatment of the most common symptoms. OBJECTIVE: to identify the knowledge that has the community nurses about the cares in the Alzheimer diseases. METHOD: a descriptive and transversal study was carried out in 20 nursing that works at the Julio Antonio Mella Teaching Policlinic in Guanabacoa municipality, from January to March of 2018. The variables studied were: age, sex, years in the professional exercise, degree and cares to the caregiver of patients with Alzheimer diseases. The information was obtained of the knowledge questionnaire. It was used means, standard deviation, absolute frequencies, percentage and Alpha of Cronbach. RESULTS: the mean was of 46.05 years. $95.0 \%$ of the participants were of the female sex, $75.0 \%$ were Registered Nurse, with 30 yearold and more of professional exercise $(60.0 \%) ; 15.0 \%$ didn't refer cares to carry out to the caregivers of patient with Alzheimer diseases. CONCLUSIONS: the community nurses that work at the Julio Antonio Mella Teaching Policlinic present little knowledge about the cares to carry out to the caregiver of patient with Alzheimer diseases.
\end{abstract}

Keywords: dementia, Alzheimer diseases, nursing staff, nursing care, community health nursing, knowledge.

http://dx.doi.org/10.7764/Horiz_Enferm.32.2.108-117

\section{INTRODUCCIÓN}

La demencia ha sido considerada a través del tiempo como una enfermedad no transmisible progresiva que afecta diver- sas funciones superiores, por la degeneración, muerte y daño de las neuronas. Esta representa un alto costo social y 
Garzón Patterson M, Izquierdo Medina R, Pascual Cuesta Y

económico, al ser la tercera causa de mortalidad después del cáncer y, según la American Psychiatric Association es un trastorno neurocognitivo mayor. ${ }^{(1)}$

La literatura consultada plantea que las personas que sufren de Síndrome Demencial presentan una declinación progresiva de la memoria, de las funciones ejecutivas, lenguaje y de otras áreas de la cognición, asociada a síntomas conductuales, que repercuten en el normal desenvolvimiento del individuo en su medio familiar y social ${ }^{(2,3)}$.

Se estima que 46,8 millones de personas sufren de demencia a nivel mundial, con 7,6 millones de nuevos casos en el año. El número de personas con demencia se duplicará cada 20 años para alcanzar los 74,7 millones en el 2030 y 131,5 millones en el 2050, incremento que será más marcado en las regiones en vías de desarrollo que en las regiones desarrolladas. La incidencia de la demencia a nivel mundial es 9,9 millones de casos, es decir cada 3,2 segundos se diagnostica un nuevo caso ${ }^{(4)}$.

De acuerdo con Bosch Bayard et al., en Cuba no se registra la prevalencia de la demencia en el anuario estadístico, aun así, esta enfermedad constituye la sexta causa de muerte en todas las edades $^{(5,}{ }^{6)}$. No obstante, estudios epidemiológicos realizados en el país por Rodríguez Rivera et al. en los municipios de Marianao, la Lisa, Playa, 10 de Octubre, Bauta y en algunos de los cabeceras de Matanzas muestran una prevalencia del síndrome demencial entre un 6,4 y 10,2 \% en la población de 65 años y más, con un predominio en el sexo femenino y corresponde a la enfermedad de Alzheimer un 5,5\% ${ }^{(7)}$.
En Cuba el envejecimiento poblacional ha sido una preocupación del partido y el estado, por lo que se encuentra entre los 29 países de un grupo de 190 que han elaborado estrategias o planes de acción para enfrentar la demencia, pocos tienen economías en vías de desarrollo. En el año 2014, Cuba elaboró la Estrategia para la enfermedad de Alzheimer y los síndromes demenciales, que, junto con su plan de acción, se ha actualizado con periodicidad, y se implementa como parte del Programa de Atención al Adulto Mayor $^{(5)}$.

La enfermedad de Alzheimer se caracteriza por ser una afección que destruye las células del cerebro, evoluciona de manera progresiva y se asocia a una de las causas de fallecimiento del paciente en un período variable de entre 4 y 20 años $^{(3,8)}$. Las manifestaciones que se valoran como criterio diagnóstico son las alteraciones cognitivas, funcionales, conductuales y signos neurológicos clásicos ${ }^{(9)}$.

El cuadro clínico se divide en tres fases $^{(3,10)}$ :

Fase 1, leve: la persona olvida algunas cosas; comienza a perder vocabulario y le cuesta construir rápidamente frases; siente dificultad para realizar actividades que antes desarrollaba de manera mecánica; pierde la referencia espacio-temporal de forma momentánea; se alteran aspectos de la personalidad.

Fase 2, moderada: el enfermo olvida los sucesos recientes; apenas puede decir unas frases lógicas seguidas; abandona sus actividades; se pierde cuando sale a la calle o en el hogar, por lo que no puede ir solo a ningún sitio; percibe 
Conocimiento de las enfermeras comunitarias sobre cuidados...

de manera errónea el espacio; puede tener reacciones desmesuradas en su carácter.

Fase 3, severa: deriva poco a poco en la muerte del cerebro y el fallecimiento del enfermo. Es frecuente que el enfermo esté encamado o postrado en una silla, por lo que la familia no tiene que estar las 24 horas del día pendiente del afectado.

La irreversibilidad de la enfermedad, la falta de tratamiento curativo y la gran demanda de cuidados que requiere hacen que sea una enfermedad importante para la sociedad. El diagnóstico precoz y las medidas terapéuticas que eviten su aparición o frenen su evolución, así como mejorar la calidad de vida de los enfermos y de sus cuidadores son el reto actual ${ }^{(11)}$.

Para abordar la enfermedad de Alzheimer se precisa de una adecuada coordinación entre los distintos niveles asistenciales y una orientación asistencial desde un equipo interdisciplinar, que fomente la atención integral del individuo y su familia ${ }^{(9)}$. En el cual, colaboran distintos profesionales y en los que el personal de enfermería tiene una relevancia especial tanto en atención primaria como en especializada, ya que están de manera continua en contacto con el enfermo ${ }^{(11)}$ y su cuidador.

Por lo que es importante que los profesionales que intervienen en la atención y cuidado del paciente con enfermedad de Alzheimer y su cuidador dispongan de conocimientos y habilidades suficientes para su prevención, captación, diagnóstico inicial, seguimiento y tratamiento de los síntomas más comunes (9).

En Cuba la Atención Primaria de Salud (APS) tiene un papel determinante en la atención a las personas sanas y enfermas en la comunidad. El médico y la enfermera de la familia, unida al grupo básico de trabajo, son los encargados de llevar la salud al seno familiar a través de los programas creados a tales efectos. Uno de ellos está dirigido a la atención integral de las personas mayores, en el que un equipo multidisciplinario realiza labores de promoción, prevención, curación y rehabilitación de salud a este grupo poblacional $^{(10)}$.

En la investigación realizada por Pascual Cuesta et al en 2019 en el municipio Cotorro, se evidenció que las enfermeras que laboran en la comunidad no brindan suficientes cuidados a la persona con Alzheimer y a los familiares que lo cuidan ${ }^{(12)}$.

Los antecedentes y la problemática antes enunciada conducen a los autores a la siguiente interrogante: ¿Qué conocimiento tienen las enfermeras comunitarias sobre los cuidados en la enfermedad de Alzheimer?

Por lo que esta investigación tuvo como objetivo identificar los conocímientos que tienen las enfermeras comunitarias sobre los cuidados en la enfermedad de Alzheimer.

\section{METODOLOGÍA}

\section{Tipo de estudio}

Se realizó un estudio descriptivo de corte transversal en el Policlínico Docente "Julio Antonio Mella" del municipio Guanabacoa, La Habana, Cuba, durante enero y marzo del año 2018. Este centro de salud se responsabiliza con la atención integral y dispensarizada ${ }^{(13)}$, en el cual el equipo básico de salud (médico y enfermera) programa actividades que 
Garzón Patterson M, Izquierdo Medina R, Pascual Cuesta Y

tienen en cuenta el cuidado de la familia como célula fundamental de la sociedad; además de realizar acciones específicas en grupos poblacionales priorizados, ${ }^{(14)}$ entre los que se encuentra los adultos mayores.

Se selecciona esta institución de salud debido a que en estudio exploratorio realizado por Garzón Patterson se pudo constatar que el $19.4 \%$ de la población es adulta mayor, de los cuales el $1.06 \%$ padece de enfermedad de Alzheimer ${ }^{(3)}$.

\section{Universo}

El universo estuvo constituido por las 41 enfermeras (os) que forman parte del equipo básico de salud de los consultorios que pertenecen al Policlínico Docente Julio Antonio Mella.

\section{Muestra}

Se seleccionó una muestra no probabilística, intencional y por conveniencia, que quedó conformada por 20 enfermeras (os) que cumplieron como criterios de inclusión: la voluntariedad de participar en el estudio, que tuvieran dispensarizados dentro de su población pacientes con diagnóstico de enfermedad de Alzheimer en las fases leve y moderada $\mathrm{y}$, que estos pacientes tuvieran cuidadores. Se seleccionan los pacientes en las fases leve y moderada porque estos necesitan ser supervisados de manera casi constante por el cuidador.

\section{Variables de estudio}

Las variables estudiadas fueron: edad, sexo, años en el ejercicio profesional, titulación y cuidados al cuidador de pacientes con enfermedad de Alzheimer. Se consideró:
Edad: tiempo que una persona ha vivido desde su nacimiento.

Sexo: según sexo biológico.

Años en el ejercicio profesional: tiempo que lleva cumpliendo funciones como enfermera(o).

Titulación: según titulación alcanzada.

Cuidados al cuidador de pacientes con enfermedad de Alzheimer: acciones que realiza el personal de enfermería con los cuidadores de pacientes con enfermedad de Alzheimer.

\section{Métodos}

Fueron utilizados como métodos del nivel teórico la sistematización, el análisis documental y el histórico lógico. Para la aplicación del método empírico, el personal de enfermería debió responder el cuestionario de conocimiento. Este instrumento fue diseñado y validado por Vázquez Rodríguez en el 2017(15); validado y adaptado por los autores, lo cual mostró buena consistencia interna medida por un Alfa de Cronbach igual a 0,852 , que plantea la homogeneidad intraítem y por tanto se consideró de adecuado, por encontrarse el valor alfa entre 0,80 y 0,90 . Este cuestionario consta de 12 ítems el cual permite la obtención de datos clasificados en la caracterización del personal de enfermería y datos relacionados con los cuidadores de pacientes con enfermedad de Alzheimer.

\section{Análisis estadístico}

Como procedimientos estadísticos se utilizó de la Estadística descriptiva la frecuencia absoluta y porcentaje en caso de variables cualitativas $y$, media $y$ desviación típica en las cuantitativas; 
además, se empleó el coeficiente de Alpha de Cronbach para la validación de la consistencia interna del cuestionario de conocimiento. El procesamiento y análisis de los datos se realizó a través del uso del paquete estadístico IBM SPSS versión 20.0 para Windows.

\section{Consideraciones éticas}

El estudio fue aprobado por la Comisión de Ética del Consejo Científico de la Facultad de Enfermería "Lidia Doce" y del Policlínico Docente "Julio Antonio Mella”. Los participantes en la investigación fueron informados de su libertad a participar y de retirarse de la investigación en cualquier momento, si así lo estimaran pertinente, sin que su decisión afectara las relaciones con el personal a cargo de la misma; para lo se procedió con la firma del consentimiento de participación.

\section{RESULTADOS}

Los resultados obtenidos del instrumento aplicado, demuestran en la Tabla 1, según la distribución del personal de Enfermería según características sociodemográficas, que en la población estudiada predomina el sexo femenino. La media de edad fue de 46,05 y una desviación típica de 4.880, significa que el promedio el grupo de edades se ubica en 46,05 años. Así mismo se desvía de 46,05, en promedio, 4.880 unidades respecto a la media. Se evidencia que el 75,0\% del personal de Enfermería posee el título de licenciado, en tanto el 25,0\% es graduado de Técnico Medio en Enfermería.
En cuanto al ejercicio profesional el $60,0 \%$ posee 30 años y más de experiencia, el $25,0 \%$ entre 25 y 29 años y el $15,0 \%$ entre 20 y 24 años.

La Tabla 2 refleja que el personal de Enfermería consideró que los cuidados que se le brindan al cuidador del paciente con enfermedad de Alzheimer están enfocadas a informar la enfermedad y las necesidades del enfermo $(60,0 \%)$, promover la implicación familiar $(25 \%)$, educar sobre la actuación ante los problemas físicos y conductuales $(10,0 \%)$, prestar atención a la salud física y psicológica del cuidador $(10,0 \%)$, cumplir tratamiento médico $(35,0 \%)$, informar sobre la red de apoyo social (40,0\%), y $15,0 \%$ no refieren acciones.

Tabla 1. Distribución del personal de Enfermería según características sociodemográficas. Policlínico Docente "Julio Antonio Mella”. 2018.

\begin{tabular}{lcc}
\hline \multicolumn{1}{c}{ Variables } & $\begin{array}{c}\text { Frecuencia } \\
(\mathrm{n}=20)\end{array}$ & $\%$ \\
\hline $\begin{array}{l}\text { Edad; media } \\
\text { (SD) }\end{array}$ & $46,05(4.880)$ & \\
\hline Sexo & & \\
Femenino & 19 & 95,0 \\
Masculino & 1 & 5,0 \\
\hline Titulación & & \\
Licenciado en & 15 & 75,0 \\
Enfermería & & \\
Técnico Medio & 5 & 25,0 \\
\hline Ejercicio & & \\
Profesional & & \\
20 - 24 & 3 & 15,0 \\
25 - 29 & 5 & 25,0 \\
30 y más & 12 & 60,0 \\
\hline Fuente· Cuestionario de conocimiento &
\end{tabular}

Fuente: Cuestionario de conocimiento. 
Garzón Patterson M, Izquierdo Medina R, Pascual Cuesta Y

Tabla 2. Distribución de cuidados realizadas por el personal de Enfermería al cuidador del paciente con enfermedad de Alzheimer. Policlínico Docente "Julio Antonio Mella". 2018.

\begin{tabular}{|c|c|c|}
\hline $\begin{array}{l}\text { Acciones } \quad \text { de } \\
\text { Enfermería }\end{array}$ & Frecuencia & $\%$ \\
\hline $\begin{array}{ll}\text { Informar sobre la } \\
\text { enfermedad y } & \text { las } \\
\text { necesidades } & \text { del } \\
\text { enfermo } & \end{array}$ & 12 & 60,0 \\
\hline $\begin{array}{l}\text { Promover la } \\
\text { implicación } \\
\text { familiar }\end{array}$ & 5 & 25,0 \\
\hline $\begin{array}{l}\text { Educar sobre la } \\
\text { actuación ante los } \\
\text { problemas físicos y } \\
\text { conductuales }\end{array}$ & 2 & 10,0 \\
\hline $\begin{array}{l}\text { Prestar atención a la } \\
\text { salud física y } \\
\text { psicológica del } \\
\text { cuidador principal }\end{array}$ & 2 & 10,0 \\
\hline $\begin{array}{l}\text { Cumplir } \\
\text { tratamiento médico }\end{array}$ & 7 & 35,0 \\
\hline $\begin{array}{l}\text { Informar sobre la } \\
\text { red de apoyo social }\end{array}$ & 8 & 40,0 \\
\hline $\begin{array}{ll}\text { No } & \text { refieren } \\
\text { acciones } & \end{array}$ & 3 & 15,0 \\
\hline
\end{tabular}

Fuente: Cuestionario de conocimiento.

\section{DISCUSIÓN Y CONCLUSIONES}

Los resultados de las variables sexo se relacionan con los encontrados por Pascual Cuesta ${ }^{(10)}$ en investigación realizada Cuba, provincia La Habana en el municipio Cotorro; en estudio realizado en el contexto internacional se obtuvo similar resultado. ${ }^{(16)}$ La enfermería a través de los tiempos ha sido considerada una profesión de mujeres, no obstante, en la actualidad no es despreciable la cantidad de hombres que ejercen esta noble profesión.

Con relación al grupo de edades los resultados obtenidos en la presente investigación difieren con los encontrados en estudio nacional ${ }^{(10)} \mathrm{e}$ internacional ${ }^{(18)}$.

Con respecto a la variable titulación, investigación realizada por Vásquez Rodríguez ${ }^{(15)}$ en una muestra de 31 enfermeros que laboraban en el Instituto de Nefrología ubicado en La Habana, Cuba; constató que el 100,0\% eran licenciado en enfermería, en el ejercicio profesional de 11 a 25 años $(45,2 \%)$, lo cual no coincide con los resultados encontrados en el presente estudio.

Investigación realizada por Gonzalez Aguiar ${ }^{(19)}$ en el Hospital Universitario "General Calixto García" en una población de 30 profesionales de enfermería evidenció que el 46,6\% poseían experiencia entre 1 y 5 años en la atención al paciente crítico y que el 53,3\% tenían estudios universitarios como Licenciados en Enfermería.

Estudio realizado en España ${ }^{(20)}$ a 77 profesionales de enfermería: 27 en el ámbito extrahospitalario y 50 en el medio intrahospitalario, demostró que el 52\% de los profesionales del grupo del medio extrahospitalario y el $58 \%$ del grupo que pertenecía al medio intrahospitalario poseían más de 10 años de experiencia laboral; lo cual no coincide con estos resultados.

Parra-Anguita et al. ${ }^{(16)}$ en investígación realizada en 24 residencias para adultos mayores demostró una mayor presencia de enfermeras auxiliares y trabajadores al cuidado de los adultos que 
Conocimiento de las enfermeras comunitarias sobre cuidados...

enfermeras licenciadas; al tener en consideración los años de experiencias, el mayor porcentaje de los encuestados se encontraba entre 5 y 15 años.

La razón de que no exista coincidencia entre los estudios comparados y los resultados de la presente investigación pudiera estar ocasionado en que el personal de enfermería una vez graduado, en su mayoría, es ubicado en los servicios de la atención secundaria y terciaria de salud, pero al pasar de los años se trasladan para la APS, generalmente por razones personales.

Con respecto a las acciones que se le brindan al cuidador del paciente con enfermedad de Alzheimer, resultados similares se obtuvo en estudio realizado en el contex to nacional por Otero Ceballos et al con el objetivo de diseñar una guía de prácticas para Enfermería en la atención al cuidador de pacientes con demencia, en el cual sus autores identificaron que el personal de enfermería requiere conocímientos para la atención al cuidador ${ }^{(21,22)}$.

Estudio realizado por Jan Rodríguez $^{(23,24)}$, en 2011, en La Habana evidenció que los Licenciados en Enfermería que laboran en la APS no poseen los conocimientos ni las habilidades necesarias para el cuidado al adulto mayor con Alzheimer y a su cuidador.

Se concluye que las enfermeras comunitarias que laboran en el Policlínico Docente "Julio Antonio Mella" presentan escasos conocimientos sobre los cuidados a realizar al cuidador del paciente con enfermedad de Alzheimer.

A partir de estos resultados se diseñó y validó por el Consejo Científico de la Facultad de Enfermería Lidia Doce un curso de postgrado titulado: "Labor del equipo de salud con la familia y cuidador principal de pacientes con enfermedad de Alzheimer", con posterioridad se impartió con el objetivo de capacitar al personal de Enfermería sobre el tema.

\section{REFERENCIAS BIBLIOGRÁFICAS}

1. Cerquera Córdoba AM, Matajira Camacho J, Pabón Poches DK. Caracterización de una muestra de cuidadores formales de pacientes con trastorno neurocognitivo mayor en Bucaramanga. Revista Virtual Universidad Católica del Norte [serie en internet]. 2016 [citado 2020 may 13]; (47): 4-19. Disponible en: https://www.redalyc.org/pdf/1942/194 244221002.pdf

2. Llibre Rodríguez J, Gutiérrez Herrera RF. Demencias y enfermedad de Alzheimer en América Latina y el Caribe. Rev Cubana Salud Pública [serie en Internet]. 2014 [citado 2020 may 12]; 40(3): 378-87. Disponible en: http://scielo.sld.cu/scielo.php? script=s ci_pdf\&pid=S0864$34662014000300008 \& \operatorname{lng}=\mathrm{es} \& n r m=\mathrm{is}$ o\&tlng=es.

3. Garzón Patterson M. Ansiedad y depresión en cuidadores principales de pacientes con enfermedad de Alzheimer [Tesis Doctoral]. La Habana: Universidad Ciencias Médicas de La Habana; 2019.

4. Murillo-Bonilla LM. Deterioro Cognitivo Vascular. Revista de Medicina Clínica [Internet]. 2019 sep [citado 2020 sep 4]; 3(3): 137-41. Disponible en: https://medicinaclinica.org/index.php/r mc/article/download/207/279 
Garzón Patterson M, Izquierdo Medina R, Pascual Cuesta Y

5. Bosch Bayard RI, Fernández Seco AE, Llibre Rodríguez JJ, Zayas Llerena T, Hernández Ulloa E, Rodríguez Blanco AL. Cuba implementa el Plan global de acción para la demencia aprobado por la Organización Mundial de la Salud 2017. Rev haban cienc méd [Internet]. 2019 Jun [citado 2021 Jul 30]; 18(3): 529-538. Disponible en:

http://scielo.sld.cu/pdf/rhcm/v18n3/1729519X-rhcm-18-03-529.pdf

6. Ministerio de Salud Pública. Anuario estadístico de cuba. [Internet] 2019 [acceso: 16/08/2020]. Disponible en: http://www.bvscuba.sld.cu/2017/11/20 /anuario-estadistico-de-salud-de-cuba/

7. Rodríguez Rivera L, Llibre Rodríguez JJ. Práctica médica en las demencias. La Habana: Editorial Ciencias Médicas; 2010. p. 8-22.

8. Del Hoyo Manrique N. La cara oculta del Alzheimer: los cuidadores familiares. [Tesis Doctoral]. España: Universidad de Valladolit; 2015.

9. Navarro Martínez M, Jiménez Navascués L, García Manzanares MC, De Perosanz Calleja M, Blanco Tobar E. Los enfermos de Alzheimer y sus cuidadores: intervenciones de enfermería. Gerokomos [serie en Internet]. 2018 [citado 2021 Agost 01]; 29(2): 79-82. Disponible en: https://scielo.isciii.es/pdf/geroko/v29n 2/1134-928X-geroko-29-02-00079.pdf

10. Pascual Cuesta Y. Modelo de cuidados de enfermería para la familia cuidadora de la persona con Alzheimer [Tesis Doctoral]. La Habana: Universidad Ciencias Médicas de La Habana; 2021.

11. Parra Anguita L. Elaboración y validación psicométrica de una escala de conocimientos sobre cuidados de la enfermedad de Alzheimer y otras demencias. Aplicación en profesionales de residencias de mayores y en estudiantes de enfermería. [Tesis Doctoral]. España: Universidad de Jaén; 2017.

12. Pascual Cuesta Y, Aguirre Raya DA, Elers Mastrapa Y, Garzón Patterson M. Cuidado de enfermería a la familia cuidadora del adulto mayor con Alzheimer. En: XVIII Congreso de la Sociedad Cubana de Enfermería. La Habana; 2019.

13. Colectivo de autores. Enfermería familiar y social. Editorial Ciencias Médicas. La Habana, 2004.

14. Colectivo de autores. Manual de procedimiento. Enfermería familiar y comunitaria. Editorial Ciencias Médicas. La Habana, 2017.

15. Vázquez Rodríguez Y. Autocuidado de pacientes hemodializados desde su cuidador. [Tesis Doctoral]. La Habana: Universidad de Ciencias Médicas de la Habana; 2017.

16. Parra-Anguita L, García-Fernández FP, Del-Pino-Casado R, Pancorbo-Hidalgo PL. Knowledge about the Care of People with Alzheimer's disease of the Nursing Staff of Nursing Homes in Spain. Int. J. Environ. Res. Public Health [serie en Internet]. 2019 [citado 2021 Agost 01]; 16: 1-12. Disponible en: https://www.mdpi.com/16604601/16/24/4907

18. Pillajo Pachacama CN. Conocimientos sobre el Alzheimer por parte del profesional de enfermería para la atención del adulto mayor en las Unidades Asistenciales Docentes de Salud de la Pontificia Universidad Católica Del Ecuador (UADS - PUCE) 
Conocimiento de las enfermeras comunitarias sobre cuidados...

en el año 2019. [Tesis de fin de grado].

Ecuador: Pontificia Universidad Católica del Ecuador; 2019.

19. González Aguiar MA. Prevención de la úlcera por presión en pacientes críticos. [Tesis Doctoral]. La Habana: Universidad de Ciencias Médicas de la Habana; 2020.

20. Rodríguez Barrera AF. Formación del personal de enfermería sobre los cuidados para pacientes con demencia. [Tesis de fin de grado]. Tenerife: Universidad de la Laguna; 2019.

21. Otero Ceballos M, Tamarit Calderín N, Torres Esperón M, Benítez Hernández I. Guía de prácticas para enfermería en la atención a los cuidadores de pacientes con demencia. Rev Cub Medicina General Integral [Internet]. 2020 [citado 2020 nov 08]; 36(2): 2-15. Disponible en: http://scieloprueba.sld.cu/pdf/mgi/v36 n2/1561-3038-mgi-36-02-e899.pdf
22. Otero Ceballos M, Tamarit Calderín N. Enfermería, cuidadores principales y familia de pacientes con demencia. Rev Cub Enfermer [serie en Internet]. 2019 [citado 2020 nov 08]; 35(4). Disponible en:

http://www.revenfermeria.sld.cu/index .php/enf/article/view/2855/508

23. Jan Rodríguez I. Propuesta de superación dirigida a enfermeros para el cuidado del adulto mayor. Panorama Cuba y Salud, 2013; 8(especial 2):32.

24. Pascual Cuesta Y, Aguirre Raya DA, Elers Mastrapa Y, Garzón Patterson M. Percepción del cuidado de enfermería en la familia cuidadora del adulto mayor con Alzheimer. VI Simposio Científico Cuidar conciencia2020. Disponible en: http://cuidarconciencia2020.sld.cu/ind ex.php/cuidarconciencia/2020/paper/vi ew/67/105 Doi:10.5944/educXX1.14476. 